Kragujevac Journal of Mathematics

Volume 38(1) (2014), Pages 173-183.

\title{
THE HARMONIC INDEX OF UNICYCLIC GRAPHS WITH GIVEN MATCHING NUMBER
}

\author{
JIAN-BO LV ${ }^{1}$, JIANXI LI ${ }^{1}$, AND WAI CHEE SHIU ${ }^{2}$
}

\begin{abstract}
The harmonic index of a graph $G$ is defined as the sum of weights $\frac{2}{d(u)+d(v)}$ of all edges $u v$ of $G$, where $d(u)$ and $d(v)$ are the degrees of the vertices $u$ and $v$ in $G$, respectively. In this paper, we determine the graph with minimum harmonic index among all unicyclic graphs with a perfect matching. Moreover, the graph with minimum harmonic index among all unicyclic graphs with a given matching number is also determined.
\end{abstract}

\section{INTRODUCTION}

Let $G$ be a simple connected graph with vertex set $V(G)$ and edge set $E(G)$. Its order is $|V(G)|$, denoted by $n$. For $v \in V(G)$, let $d_{G}(v)$ (or $\left.d(v)\right)$ and $N_{G}(v)($ or $N(v)$ ) be the degree and the neighborhood of $v$, respectively.

The Randic index is one of the most successful molecular descriptors in structureproperty and structure-activity relationships studies. The Randić index of a graph $G$ is defined in [13] as the sum of the weights $(d(u) d(v))^{-\frac{1}{2}}$ over all edges $u v$ of $G$. The mathematical properties of this graph invariant have been studied extensively (see recent book [7] and survey [9]). Motivated by the success of Randić index, various generalizations and modifications were introduced, such as the sum-connectivity index $[15,17]$ and the general sum-connectivity index $[2,3]$.

Another variant of the Randic index, named the harmonic index $H(G)$, which is defined as

$$
H(G)=\sum_{u v \in E(G)} \frac{2}{d(u)+d(v)},
$$

where the summation goes over all edges $u v$ of $G$. This index was first appeared in [5]. Estimating bounds for $H(G)$ is of great interest, and many results have been obtained.

Key words and phrases. Harmonic index, Matching number, Unicyclic graph. 2010 Mathematics Subject Classification. Primary: 05C12. Secondary: 92E10.

Received: August 4, 2013. 
For example, Favaron et al. [6] considered the relationship between the harmonic index and the eigenvalues of graphs; Zhong [18, 19, 20] determined the minimum and maximum values of the harmonic index for simple connected graphs, trees, unicyclic graphs and bicyclic graphs, and characterized the corresponding extremal graphs, respectively. Li and Shiu [10] studied how the harmonic index behaves when the graph is under perturbations and provided a simpler method for determining the unicyclic graphs with maximum and minimum harmonic index among all unicyclic graphs, respectively. Moreover, lower bounds for harmonic index are also obtained in [10] and [14], respectively. Recently, Deng et al. [4] studied the relationship between the harmonic index and the chromatic number of a graph $G$, and obtained the lower bound for $H(G)$ in terms of its chromatic number. Lv and $\mathrm{Li}$ [11] studied the relationship between the harmonic index and the matching number for trees, and determined the trees with minimum harmonic index among trees with a perfect matching and among trees with a given matching number, respectively.

In this paper, we further consider the relationship between the harmonic index and the matching number for unicyclic graphs. The graph with minimum harmonic index among all unicyclic graphs with a perfect matching is determined. Moreover, the graph with minimum harmonic index among all unicyclic graphs with a given matching number is also determined.

\section{Preliminaries}

We first introduce some terminologies and notations of graphs. For a connected graph $G$, a pendant vertex is a vertex of degree 1 in $G$. Let $P V$ be the set of all pendant vertices of $G$. Let $d_{G}(x, y)$ be the length of a shortest $(x, y)$-path in $G$. We use $G-x$ to denote the graph that arises from $G$ by deleting the vertex $x \in V(G)$ together with its incident edges. An edge $e$ of $G$ is said to be contracted if it is deleted and its ends are identified; the resulting graph is denoted by $G \cdot e$. A subset $M \subseteq E$ is called a matching in $G$ if its elements are edges and any two of them are not adjacent in $G$. A matching $M$ saturates a vertex $v$, and $v$ is said to be $M$-saturated, if some edge of $M$ is incident with $v$. If every vertex of $G$ is $M$-saturated, then the matching $M$ is perfect. A matching $M$ is said to be an $m$-matching, if $|M|=m$ and for every matching $M^{\prime}$ in $G,\left|M^{\prime}\right| \leq m$.

Denote by $C_{n}$ the cycle of order $n$. Let $n$ and $m$ be positive integers with $n \geq 2 m$. Let $\mathscr{U}_{n, m}$ be the set of unicyclic graphs of order $n$ with an $m$-matching. For any $G \in \mathscr{U}_{n, m} \backslash\left\{C_{n}\right\}, G$ consists of a unique cycle, denoted by $C_{g}$, and some trees attached to some vertices on the cycle. Those vertices attached to trees, for convenience, are called the roots of the trees attached to them. A root may have more than one tree attached to it. In order to prove our main results, the following lemmas are needed.

Lemma 2.1. [1] Let $G \in \mathscr{U}_{2 m, m}(m \geq 3)$, and $T$ be a tree in $G$ attached to a root $r$. If $v \in V(T)$ is a vertex furthest from the root $r$ with $d_{G}(v, r) \geq 2$, then $v$ is a pendant vertex and adjacent to a vertex $u$ of degree 2 . 
Lemma 2.2. [12] Let $G \in \mathscr{U}_{2 m, m}$. If $P V \neq \emptyset$, then for any $u \in V(G)$, we have $|N(u) \cap P V| \leq 1$.

Lemma 2.3. [16] Let $G \in \mathscr{U}_{n, m}(n>2 m)$ and $G ¥ C_{n}$. Then there is an m-matching $M$ and a pendant vertex $v$ such that $M$ does not saturate $v$.

Lemma 2.4. (1) For $x \geq 2$, the function $f_{1}(x)=\frac{2 x-2}{x+2}-\frac{2 x-6}{x+1}-\frac{2}{x}$ is monotonicly decreasing on $x$.

(2) For $x \geq 2$, the function $f_{2}(x)=\frac{2 x}{x+2}-\frac{2 x-2}{x+1}$ is monotonicly decreasing on $x$.

(3) For $x \geq 2$, the function $f_{3}(x)=\frac{2 x-2}{x+2}-\frac{2 x-4}{x+1}$ is monotonicly decreasing on $x$.

(4) For $x \geq 3$, the function $f_{4}(x)=\frac{2}{x+1}+\frac{2 x-4}{x+2}-\frac{2 x}{x+3}$ is monotonicly increasing on $x$.

Proof. (1) We consider the derivative of $f_{1}(x)$. Note that, for $x \geq 2$, we have

$$
\begin{aligned}
\frac{d f_{1}(x)}{d x} & =\frac{6}{(x+2)^{2}}-\frac{8}{(x+1)^{2}}+\frac{2}{x^{2}} \\
& =\frac{6 x^{2}(x+1)^{2}+2(x+2)^{2}(x+1)^{2}-8(x+2)^{2} x^{2}}{x^{2}(x+1)^{2}(x+2)^{2}} \\
& =\frac{-8 x^{3}+24 x+8}{x^{2}(x+1)^{2}(x+2)^{2}}<0 .
\end{aligned}
$$

Then $f_{1}(x)$ is monotonicly decreasing on $x$.

(2) The results follows from

$$
\frac{d f_{2}(x)}{d x}=\frac{4}{(x+2)^{2}}-\frac{4}{(x+1)^{2}}<0 .
$$

(3) The results follows from

$$
\frac{d f_{3}(x)}{d x}=\frac{6}{(x+2)^{2}}-\frac{6}{(x+1)^{2}}<0 .
$$

(4) Note that, for $x \geq 3$, we have

$$
\begin{aligned}
\frac{d f_{4}(x)}{d x} & =\frac{-2}{(x+1)^{2}}+\frac{8}{(x+2)^{2}}+\frac{-6}{(x+3)^{2}} \\
& =\frac{8(x+1)^{2}(x+3)^{2}-2(x+2)^{2}(x+3)^{2}-6(x+1)^{2}(x+2)^{2}}{(x+1)^{2}(x+2)^{2}(x+3)^{2}} \\
& =\frac{8(x+1)^{3}-24(x+1)-8}{(x+1)^{2}(x+2)^{2}(x+3)^{2}}>0 .
\end{aligned}
$$

Thus the result holds.

Lemma 2.5. Let $x, y$ be positive integers with $1 \leq y \leq x-1$. Let

$$
g(x, y)=\frac{2 x-2 y}{x+2}-\frac{2 x-4 y}{x+1}-\frac{2 y-2}{x} .
$$

Then $g(x, y)$ is monotonicly decreasing on $x$ and $y$, respectively. 
Proof. We consider some partial derivatives of $g(x, y)$. Note that

$$
\begin{aligned}
\frac{\partial g(x, y)}{\partial y} & =\frac{4}{x+1}-\frac{2}{x+2}-\frac{2}{x} \\
& =\frac{-4}{(x+2)(x+1) x}<0 .
\end{aligned}
$$

Then $g(x, y)$ is monotonicly decreasing on $y$.

On the other hand,

$$
\begin{aligned}
\frac{\partial g(x, y)}{\partial x} & =\frac{2 y+4}{(x+2)^{2}}+\frac{2 y-2}{x^{2}}-\frac{4 y+2}{(x+1)^{2}} \\
& =\frac{12 x^{2} y+24 x y+8 y-12 x^{3}-30 x^{2}-24 x-8}{(x+2)^{2}(x+1)^{2} x^{2}} .
\end{aligned}
$$

Note that $y \leq x-1$. Then we have

$$
\begin{aligned}
\frac{\partial g(x, y)}{\partial x} & \leq \frac{12 x^{2}(x-1)+24 x(x-1)+8(x-1)-12 x^{3}-30 x^{2}-24 x-8}{(x+2)^{2}(x+1)^{2} x^{2}} \\
& =\frac{-18 x^{2}-40 x-16}{(x+2)^{2}(x+1)^{2} x^{2}}<0 .
\end{aligned}
$$

Thus $g(x, y)$ is monotonicly decreasing on $x$.

Let $U_{n, m}$ (Shown in Figure 1 ) be a graph of order $n$ obtained from $C_{3}$ by attaching $n-2 m+1$ pendent edges and $m-2$ paths of length 2 to one vertex of $C_{3}$. Let $H\left(U_{n, m}\right)=\Psi(n, m)$, where

$$
\Psi(n, m)=\frac{2 m}{n-m+3}+\frac{2(n-2 m+1)}{n-m+2}+\frac{2 m}{3}-\frac{5}{6} .
$$
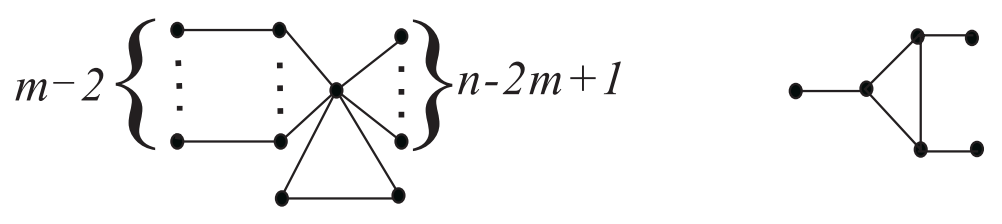

$$
U_{n, m}
$$

$H_{6}$

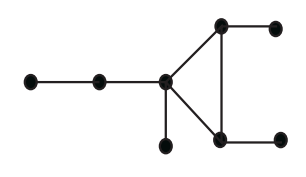

$H_{8}$

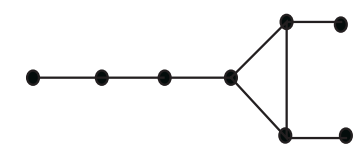

$Q_{8}$

Figure 1. Graphs $U_{n, m}, H_{6}, H_{8}$ and $Q_{8}$ 
Lemma 2.6. For $m \geq 3$, then we have

$$
\Psi(2(m-1), m-1)+\frac{4}{5}>\Psi(2 m, m)
$$

Proof. By Lemma 2.4(4), we have

$$
\begin{aligned}
& \Psi(2(m-1), m-1)+\frac{4}{5}-\Psi(2 m, m) \\
= & \frac{2}{m+1}+\frac{2 m-4}{m+2}-\frac{2 m}{m+3}+\frac{2}{15} \\
\geq & \frac{1}{2}+\frac{2}{5}-1+\frac{2}{15}>0 .
\end{aligned}
$$

Hence the result holds.

Lemma $2.7([19])$. Let $\mathscr{U}(n)$ be the set of unicyclic graphs of order $n \geq 4$. Then for any $G \in \mathscr{U}(n), H(G) \leq \frac{n}{2}$, and the equality holds if and only if $G \cong C_{n}$.

\section{MAin Results}

Now, we give the main results of this section.

Theorem 3.1. Let $G \in \mathscr{U}_{2 m, m} \backslash\left\{H_{6}, H_{8}\right\}$, where $m \geq 2$. Then $H(G) \geq \Psi(2 m, m)$, and the equality holds if and only if $G \cong U_{2 m, m}$.

Proof. Note that if $m=2$, then either $G \cong C_{4}$ or $G \cong U_{4,2}$. Then the result follows since $H\left(C_{4}\right)=2>H\left(U_{4,2}\right)=\frac{9}{5}$.

So, we suppose that $m \geq 3$. We now prove the assertion by the induction on $m$. If $G \cong C_{2 m}$, then Lemma 2.7 implies that $H\left(C_{2 m}\right)>\Psi(2 m, m)$. The result follows. In what follows, we assume that $G \nsubseteq C_{2 m}$. From Lemmas 2.1 and 2.2, we need only consider the following two cases.

Case $1 \quad G$ has a pendant vertex $v$ such that $N(v)=\{w\}$ and $d(w)=2$.

In this case, there is a unique vertex $u \neq v$ such that $u w \in E(G)$. Let $N(u) \cap P V=$ $\left\{v_{1}, v_{2}, \cdots, v_{r-1}, v_{r}\right\}$ and $N(u) \backslash P V=\left\{x_{1}, \cdots, x_{t-r-1}, x_{t-r}=w\right\}$. Then $t \leq m+1$, $d\left(x_{j}\right) \geq 2$ for each $j=1, \ldots, t-r-1$ and $d\left(x_{t-r}\right)=d(w)=2$. Let $G^{\prime}=G-v-w$. Clearly, $G^{\prime} \in \mathscr{U}_{2(m-1), m-1}$.

If $G^{\prime} \cong H_{6}$, then $G \cong Q_{8}$, where $H_{6}$ and $Q_{8}$ are shown in Figure 1 . Hence the result follows since $H\left(Q_{8}\right)=3.5666>\Psi(8,4)=3.3095$.

If $G^{\prime} \cong H_{8}$ (shown in Figure 1), then $G \in\left\{G_{i} \mid 1 \leq i \leq 6\right\}$, where $G_{i}(1 \leq i \leq 6)$ and their harmonic indices are shown in Figure 2, respectively. Hence the result follows since $H\left(G_{i}\right)>\Psi(10,5)=4.0357$ for $1 \leq i \leq 6$. 


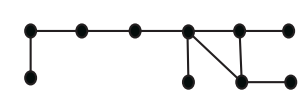

4.3047

$G_{1}$

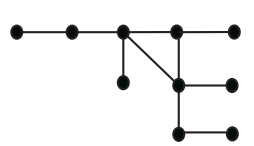

4.1214

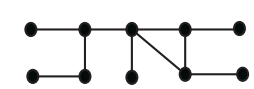

4.1571

$G_{2}$

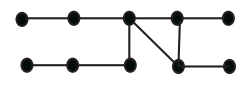

4.4047

Gs

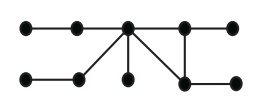

4.0714

G3

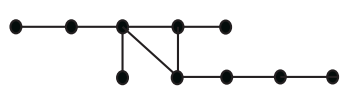

4.3714

G。

Figure 2. Graphs $G_{1}, \ldots, G_{6}$ and their harmonic indices

If $G^{\prime} \notin\left\{H_{6}, H_{8}\right\}$, then by the induction hypothesis, we have

$$
\begin{aligned}
H(G)= & H\left(G^{\prime}\right)+\frac{2}{3}+\frac{2}{t+2}+r\left(\frac{2}{t+1}-\frac{2}{t}\right) \\
& +\sum_{i=1}^{t-r-1}\left(\frac{2}{t+d\left(x_{i}\right)}-\frac{2}{t+d\left(x_{i}\right)-1}\right) \\
\geq & H\left(G^{\prime}\right)+\frac{2}{3}+\frac{2}{t+2}+r\left(\frac{2}{t+1}-\frac{2}{t}\right) \\
& +(t-r-1)\left(\frac{2}{t+2}-\frac{2}{t+1}\right) \\
\geq & \Psi(2 m-2, m-1)+\frac{2}{3}+\frac{2}{t+2}+r\left(\frac{2}{t+1}-\frac{2}{t}\right) \\
& +(t-r-1)\left(\frac{2}{t+2}-\frac{2}{t+1}\right) \\
= & \Psi(2 m, m)+\frac{2}{m+1}+\frac{2 m-4}{m+2}-\frac{2 m}{m+3} \\
& +\frac{2}{t+2}+r\left(\frac{2}{t+1}-\frac{2}{t}\right)+(t-r-1)\left(\frac{2}{t+2}-\frac{2}{t+1}\right) .
\end{aligned}
$$

Note that by Lemma 2.2, we have $r \leq 1$. Recall that $t \leq m+1$.

If $r=1$, then by Lemma 2.4(1), we have 


$$
\begin{aligned}
H(G) \geq & \Psi(2 m, m)+\frac{2}{m+1}+\frac{2 m-4}{m+2}-\frac{2 m}{m+3} \\
& +\frac{2 t-2}{t+2}-\frac{2 t-6}{t+1}-\frac{2}{t} \\
\geq & \Psi(2 m, m)+\frac{2}{m+1}+\frac{2 m-4}{m+2}-\frac{2 m}{m+3} \\
& +\frac{2 m}{m+3}-\frac{2 m-4}{m+2}-\frac{2}{m+1} \\
= & \Psi(2 m, m) .
\end{aligned}
$$

Suppose that the equality holds. Then all inequalities in the above argument should be equalities.

Then we have $H\left(G^{\prime}\right)=\Psi(2 m-2, m-1), t=m+1$ and $d\left(x_{1}\right)=\cdots d\left(x_{t-r}\right)=2$. Thus by the induction hypothesis, we have $G^{\prime} \cong U_{2 m-2, m-1}$. Hence $G \cong U_{2 m, m}$.

If $r=0$, then by Lemma 2.4(2), we have

$$
\begin{aligned}
H(G) \geq & \Psi(2 m, m)+\frac{2}{m+1}+\frac{2 m-4}{m+2}-\frac{2 m}{m+3} \\
& +\frac{2}{t+2}+(t-1)\left(\frac{2}{t+2}-\frac{2}{t+1}\right) \\
= & \Psi(2 m, m)+\frac{2}{m+1}+\frac{2 m-4}{m+2}-\frac{2 m}{m+3} \\
& +\frac{2 t}{t+2}-\frac{2 t-2}{t+1} \\
\geq & \Psi(2 m, m)+\frac{2}{m+1}-\frac{4}{m+2}+\frac{2}{m+3} \\
= & \Psi(2 m, m)+\frac{4}{(m+1)(m+2)(m+3)} \\
> & \Psi(2 m, m) .
\end{aligned}
$$

Case $2 G$ is a cycle $C_{g}$ together with some pendant edges attached to some vertices on $C_{g}$. For convenience, we label the vertices of $C_{g}$ with $u_{1}, u_{2}, \cdots u_{g}$.

Note that $G \varsubsetneqq C_{n}$. If $d_{G}\left(u_{i}\right)=3$ for each $i=1,2, \ldots, g$, then $m \geq 4$ since $G \supsetneqq H_{6}$. If $m=4$, then the result follows since $H(G)=4\left(\frac{1}{3}+\frac{1}{2}\right)=3.3333>\Psi(8,4)=3.3095$. If $m \geq 5$, by the induction hypothesis and Lemma 2.6 , then we have

$$
\begin{aligned}
H(G)=(m-1)\left(\frac{1}{3}+\frac{1}{2}\right)+\frac{1}{3}+\frac{1}{2} & >\Psi(2 m-2, m-1)+\frac{5}{6} \\
& >\Psi(2 m-2, m-1)+\frac{4}{5} \\
& >\Psi(2 m, m) .
\end{aligned}
$$


If there exists some $i \in\{1,2, \cdots, g\}$ such that $d_{G}\left(u_{i}\right)=3$ and $d_{G}\left(u_{i+1}\right)=2$, where $u_{g+1}=u_{1}$. Without loss of generality, we assume that $d_{G}\left(u_{2}\right)=3$ and $d_{G}\left(u_{3}\right)=2$. Let $v_{2}$ the pendant vertex which adjacent to $u_{2}$. Note that every pair of vertices of degree 3 can not be adjacent to a common vertex of degree 2 since $G$ has a perfect matching. Then each vertex of degree 2 on $C_{g}$ must be adjacent to another vertex of degree 2. Thus $d_{G}\left(u_{4}\right)=2$. Let $G^{\prime}=\left(G \cdot u_{2} v_{2}\right) \cdot u_{2} u_{3}$ be a graph obtained from $G$ by contracting $u_{2} v_{2}$ and $u_{2} u_{3}$ consecutively. Clearly, $G^{\prime} \in \mathscr{U}_{n-2, m-1} \backslash\left\{H_{6}, H_{8}\right\}$. Hence, by the induction hypothesis and Lemma 2.6 , if $d_{G}\left(u_{1}\right)=3$, then

$$
\begin{aligned}
H(G)=H\left(G^{\prime}\right)+\frac{1}{3}+\frac{1}{2}+\frac{2}{5}-\frac{2}{5} & \geq \Psi(2 m-2, m-1)+\frac{1}{3}+\frac{1}{2} \\
& >\Psi(2 m, m)
\end{aligned}
$$

if $d_{G}\left(u_{1}\right)=2$, then

$$
\begin{aligned}
H(G)=H\left(G^{\prime}\right)+\frac{4}{5}+\frac{1}{2}-\frac{1}{2} & \geq \Psi(2 m-2, m-1)+\frac{4}{5} \\
& >\Psi(2 m, m) .
\end{aligned}
$$

Conversely, if $G \cong U_{2 m, m}$, then it is easy to check that the equality holds. This completes the proof.

Remark 3.1. It is easy to calculate that $H\left(H_{6}\right)=2.5<\Psi(6,3)=2.5666$ and $H\left(H_{8}\right)=$ $3.3047<\Psi(8,4)=3.3095$, where $H_{6}$ and $H_{8}$ are shown in Figure 1 . Thus, by Theorem 3.1, $H_{6}$ has the minimum harmonic index in $\mathscr{U}_{6,3}$ and $H_{8}$ has the minimum harmonic index in $\mathscr{U}_{8,4}$.

Theorem 3.2. Let $G \in \mathscr{U}_{n, m}$, where $n \geq 2 m$ and $m \geq 5$. Then $H(G) \geq \Psi(n, m)$, and the equality holds if and only if $G \cong U_{n, m}$.

Proof. We prove the assertion by the induction on $n$.

If $n=2 m$, then the result follows from Theorem 3.1. Now, we assume that $n>2 m$. If $G \cong C_{n}$, then $n=2 m+1$. Lemma 2.7 implies that $H\left(C_{2 m+1}\right)>\Psi(2 m+1, m)$. The result follows. So in what follows, we assume that $G \nsubseteq C_{n}$. Then Lemma 2.3 implies that $G$ has an $m$-matching $M$ and a pendant vertex $v$ such that $M$ does not saturate $v$. Let $u v \in E(G)$ and $d(u)=t$. Denote $N(u) \cap P V=\left\{v_{1}, \cdots, v_{r-1}, v_{r}=v\right\}$ and $N(u) \backslash P V=\left\{x_{1}, \cdots, x_{t-r}\right\}$. Then $d\left(x_{j}\right) \geq 2$ for each $j=1, \ldots, t-r$. Let $G^{\prime}=G-v$. Then $G^{\prime} \in \mathscr{U}_{n-1, m}$. Now we consider the following two cases.

Case $1 r=1$, that is $v_{1}=v$.

Note that $t \leq n-m+1$. Then by the induction hypothesis and Lemma 2.4(3), we have 


$$
\begin{aligned}
H(G)= & H\left(G^{\prime}\right)+\frac{2}{t+1}+\sum_{j=1}^{t-1}\left(\frac{2}{d\left(x_{j}\right)+t}-\frac{2}{d\left(x_{j}\right)+t-1}\right) \\
\geq & \Psi(n-1, m)+\frac{2}{t+1}+(t-1)\left(\frac{2}{2+t}-\frac{2}{1+t}\right) \\
= & \Psi(n-1, m)+\frac{2 t-2}{t+2}-\frac{2 t-4}{t+1} \\
= & \Psi(n, m)+\frac{2 n-4 m}{n-m+1}-\frac{2 n-6 m+2}{n-m+2}-\frac{2 m}{n-m+3} \\
& +\frac{2 t-2}{t+2}-\frac{2 t-4}{t+1} \\
\geq & \Psi(n, m)+\frac{2 n-4 m}{n-m+1}-\frac{4 n-8 m}{n-m+2}+\frac{2 n-4 m}{n-m+3} \\
> & \Psi(n, m) .
\end{aligned}
$$

Case $2 r \geq 2$.

Note that $t \leq n-m+1$ and $r \leq n-2 m+1$. Then by the induction hypothesis and Lemma 2.5, we have

$$
\begin{aligned}
H(G)= & H\left(G^{\prime}\right)+\frac{2}{t+1}+(r-1)\left(\frac{2}{t+1}-\frac{2}{t}\right) \\
& +\sum_{j=1}^{t-r}\left(\frac{2}{d\left(x_{j}\right)+t}-\frac{2}{d\left(x_{j}\right)+t-1}\right) \\
\geq & \Psi(n-1, m)+\frac{2 r}{t+1}-\frac{2 r-2}{t}+\sum_{j=1}^{t-r}\left(\frac{2}{d\left(x_{j}\right)+t}-\frac{2}{d\left(x_{j}\right)+t-1}\right) \\
\geq & \Psi(n-1, m)+\frac{2 r}{t+1}-\frac{2 r-2}{t}+(t-r)\left(\frac{2}{2+t}-\frac{2}{1+t}\right) \\
= & \Psi(n, m)+\frac{2 n-4 m}{n-m+1}-\frac{2 n-6 m+2}{n-m+2}-\frac{2 m}{n-m+3} \\
& -\frac{2 r-2}{t}-\frac{2 t-4 r}{t+1}+\frac{2 t-2 r}{t+2} \\
\geq & \Psi(n, m)+\frac{2 n-4 m}{n-m+1}-\frac{2 n-6 m+2}{n-m+2}-\frac{2 m}{n-m+3} \\
& -\frac{2 n-4 m}{n-m+1}+\frac{2 n-6 m+2}{n-m+2}+\frac{2 m}{n-m+3} \\
= & \Psi(n, m) .
\end{aligned}
$$

Suppose that the equality holds. Then all inequalities in the above argument should be equalities. Then we have $H\left(G^{\prime}\right)=\Psi(n-1, m), t=n-m+1, r=n-2 m+1$ and 
$d\left(x_{1}\right)=\cdots=d\left(x_{t-r}\right)=2$. Thus by the induction hypothesis, we have $G^{\prime} \cong U_{n-1, m}$. Hence, it is not difficult to see that $G \cong U_{n, m}$.

Conversely, if $G \cong U_{n, m}$, then it is easy to see that the equality holds. This completes the proof.

Acknowledgment: The authors would like to thank the anonymous referees for their constructive corrections and valuable comments on this paper, which have considerably improved the presentation of this paper. This research was partially supported by NSF of China(Nos. 11101358, 61379021); NSF of Fujian(Nos. 2011J05014, 2011J01026, 2012D140); Project of Fujian Education Department(No. JA11165, JA12208, JA12209); General Research Fund of Hong Kong; FRG of Hong Kong Baptist University.

\section{REFERENCES}

[1] A. Chang, F. Tian, On the spectral radius of unicyclic graphs with perfect matchings, Lin. Alg. Appl. 370 (2003), 237-250.

[2] Z. Du, B. Zhou, N. Trinajstić, Minimum general sum-connectivity index of unicyclic graphs, J. Math. Chem. 48 (2010) 697-703.

[3] Z. Du, B. Zhou, N. Trinajstić, On the general sum-connectivity index of trees, Appl. Math. Lett. 24 (2011), 402-405.

[4] H. Deng, S. Balachandran, S.K. Ayyaswamy, Y.B. Venkatakrishnan, On the harmonic index and the chromatic number of a graph, Discrete Appl. Math. 161 (2013) 2740-2744.

[5] S. Fajtlowicz, On conjectures of GraffitiII, Congr. Numer. 60 (1987), 187-197.

[6] O. Favaron, M. Mahéo, J. F. Saclé, Some eigenvalue properties in graphs(conjectures of GraffitiII), Discrete Math. 111 (1993), 197-220.

[7] I. Gutman, B. Furtula (Eds.), Recent Results in the Theory of Randic Index, Mathematical Chemistry Monographs 6, University of Kragujevac, 2008.

[8] Y. Hou, J. Li, Bounds on the largest eigenvalues of trees with a given size of matching, Lin. Alg. Appl. 342 (2002), 203-217.

[9] X. Li, Y. Shi, A survey on the Randić index, MATCH Commun. Math. Comput. Chem. 59 (2008), 127-156.

[10] J. Li, W. C. Shiu, The harmonic index of a graph, Rocky Mountain J. Math. to appear.

[11] J.-B. Lv, J. Li, On the harmonic index and the matching number of a tree, Ars Combin. to appear.

[12] X. Pan, H. Liu, J. Xu, Sharp lower bounds for the general Randic index of trees with a given size of matching, MATCH Commun. Math. Comput. Chem. 54 (2005), 465-480.

[13] M. Randić, On characterization of molecular branching, J. Am. Chem. Soc. 97 (1975) 66096615.

[14] R. Wu, Z. Tang, H. Deng, A lower bound for the harmonic index of a graph with minimum degree at least two, Filomat, 27 (2013), 51-55.

[15] R. Xing, B. Zhou, N. Trinajstić, Sum-connectivity index of molecular trees, J. Math. Chem. 48 (2010), 583-591.

[16] A. Yu, F. Tian, On the spectral radius of unicyclic graphs, MATCH Commun. Math. Comput. Chem. 51 (2004), 97-109.

[17] B. Zhou, N. Trinajstić, On a novel connectivity index, J. Math. Chem. 46 (2009), 1252-1270.

[18] L. Zhong, The harmonic index for graphs, Appl. Math. Lett. 25 (2012), 561-566.

[19] L. Zhong, The harmonic index on unicyclic graphs, Ars Combin. 104 (2012), 261-269.

[20] L. Zhong, K. Xu, The harmonic index for bicyclic graphs, Utilitas Math. 90 (2013), 23-32. 
THE HARMONIC INDEX OF UNICYCLIC GRAPHS WITH GIVEN MATCHING NUMBER 183

${ }^{1}$ School of Mathematics and Statistics,

Minnan Normal University, Zhangzhou, Fujian, P. R. China

E-mail address: ptjxli@hotmail.com (J. Li, Corresponding author)

${ }^{2}$ Department of Mathematics,

Hong Kong Baptist University, Kowloon Tong, Hong Kong, P.R. China.

E-mail address: wcshiu@hkbu.edu.hk 